\title{
Validation of a Model of a Railway Vehicle Based on CFD Function Comparison
}

\author{
Nebojša Bogojević ${ }^{1 *}$, Jelena Tomić ${ }^{1}$, Slobodan Todosijević ${ }^{1}$ \\ ${ }^{1}$ Faculty of Mechanical and Civil Engineering Kraljevo, University of Kragujevac, Kraljevo (Serbia)
}

Recent improvements of computers and numerical tools gave engineers a possibility to develop and use very complex numerical models. One of the essential steps in the model developing is a process of verification and validation. Traditionally, comparisons of time histories on a graph have been used to make subjective engineering judgments, how well the histories agree or disagree. In order to introduce an objective assessment of agreement between simulation and experimental results of two validation metrics were presented in this paper. The Area validation metrics and KolmogorovSmirnov test, based on the comparison of the cumulative probability functions, were used for validation of developed model of a rail vehicle.The obtained results have shown that presented validation metrics may be used for the model validation. However, the subjective judgment in the process of the validation cannot be completely avoided. The future work should be focused on development of a new validation metric which will eliminate subjective judgment in the process of the model validation.

\section{Keywords: Validation, Rail vehicle, Model, Simulation}

\section{INTRODUCTION}

Experimental investigation of railway vehicles is the most reliable way to determine their properties and the crucial criterion for the approval of their exploitation. However, experimental investigations are extensive, timeconsuming and expensive so alternative methods, used in the design of vehicles are of greatest interest. Numerical simulations of the railway vehicle running behaviour, which allows the calculation of dynamical quantities in the time and frequency domain based on the mathematical models of the vehicle and track, are developed in that purpose.

In the field of the railway vehicle dynamics the simulations of the vehicle behaviour are used for:

- Design and development of the vehicle in all stages,

- Predict vehicle behaviour in various exploitation conditions,

- To determine compliance with the requirements of running security and safety of the vehicle,

- Analyse how modification in vehicles will affect on the dynamic behaviour of the vehicle,

- Analysis of the vehicle behaviour in the cases when it's not possible to perform experimental investigation.

Taking into account that numerical models are used in all stages of the vehicle design and development, it is necessary to develop a methodology to assess how much we may rely on the results obtained from simulations.

The process which determines or perform evaluation of agreement of the experimental results with the results obtained by numerical simulation is called the process of model validation and verification.

The model validation is the process where is possible to determine the degree that a model is an accurate representation of the real system [2], [3].

The verification process is focused on the identification and elimination of errors in the development of mathematical and computer models [1]. While the mathematical model comprises the conceptual model, mathematical equations, and modeling data needed to describe real system [1], [2], [3], the computer model represents encapsulation of the mathematical model in the form suitable for execution on a computer [1].

The methodology for evaluation of the agreement between the results obtained by simulation and experimental investigation has not been defined in the field of the railway vehicle dynamics. The model validation, applied from different authors [4], [5], [6], [7], [8] was performed by comparing the characteristic parameters, such as accelerations in vertical and horizontal plane and forces in the wheel-rail contact, in time and/or frequency domain.

In general the model validation may be performed using five different approaches:

- Graphical comparison of different diagrams,

- Comparison of the characteristic features of the signals,

- Comparison of the CDF (cumulative density functions) - PDF (probability density functions) functions

- Perform a hypothesis testing and

- Validation based on the Bayesian framework

Graphical methods are based on the comparison of various graphs. The results of simulations are plotted together with the results from experiments on the same graph. This method does not provide quantitative measure of matching between the results obtained by simulations and experiments. The model validation performed by this method is highly subjective and depends on the experience of the reviewer. In the field of railway vehicle dynamics, graphical comparison of different parameters is the most common method used for model validation.

Feature-based techniques draw conclusions on the model validation based on the difference between characteristic features of the obtained results, such as magnitude, shape, phase, etc. Various metrics are used as a 
measure of the difference. One of the most known metrics is defined by Sprague and Geer [9], and it is based on the difference between magnitudes and phases of the results of simulations and experiments. The Russell metrics [10,11] are very similar to the SG metric. EARTH metrics [12] take into account the shape of scalar series, which is not the case with SG and Russell metrics.

Model validation based on PDF (the probability density function) or CDF (the cumulative density function) techniques draw conclusions based on the difference between PDF or CDF functions of the obtained results. During the last fifty years, researchers have developed several validation metrics for comparison of PDF/CDF functions. The Kolmogorov-Smirnov metric [13] is one of the most used metrics for model validation. It measures the distance between two CDF functions along the ordinate axis. Anderson-Darling [14] validation metric is very similar to Kolmogorov-Smirnov metric. However, instead the distance along ordinate, Anderson-Darling metric has introduced the weighted quadratic CDF statistic to measure the distance between the two CDF functions. It was shown that the Anderson-Darling validation statistic had more power than the Kolmogorov-Smirnov metrics [13]. The third validation metrics - Area validation metrics is based on the calculation of the area between the two CDF functions [15]. The area metrics depend on the scale used to present the distributions, and any kind of normalization would destroy the meaning of the metrics [18].

This paper presents the model validation of the freight railway vehicle with three piece bogie "Motion Control M976 Truck System” of Amsted rail Co, by comparing CDF functions of the accelerations on the carbody floor, vertical and lateral forces in the wheel-rail contact.

\section{THE MODEL OF THE VEHICLE WITH THREE PIECE BOGIE}

Freight railway vehicle with three piece bogie consist of carbody for load transportation and two three piece bogies.

The three piece bogie has relatively simple design. Basic parts of the bogie are two side frames (1) which are connected with bolster (2), as it is shown in Figure 1. The connection between carbody and bogie has been established over center plate. Side frames are connected to wheelset (7) over elastic adapters (6).

The elastic connection between side frame and wheelset gives possibility of the wheelset to have a higher lateral movement's compared to conventional three piece bogie. This possibility for lateral motion of the wheelset gives better curving characteristics and possibility to increase the vehicle speed [8].

Despite the fact that the three-piece bogie is very simple, it is source of large number of the nonlinearity in the vehicle model. One of the main sources of the nonlinearity is suspension system which consists of the set of coil springs (5) that connect the side frames (1) and bolster (2) and second set of springs which connect the side frame (1) and wedge (4). The wedges in suspension system are sliding over bolster, from one side, and over side frame, from second side, as it is shown in Figure 1.

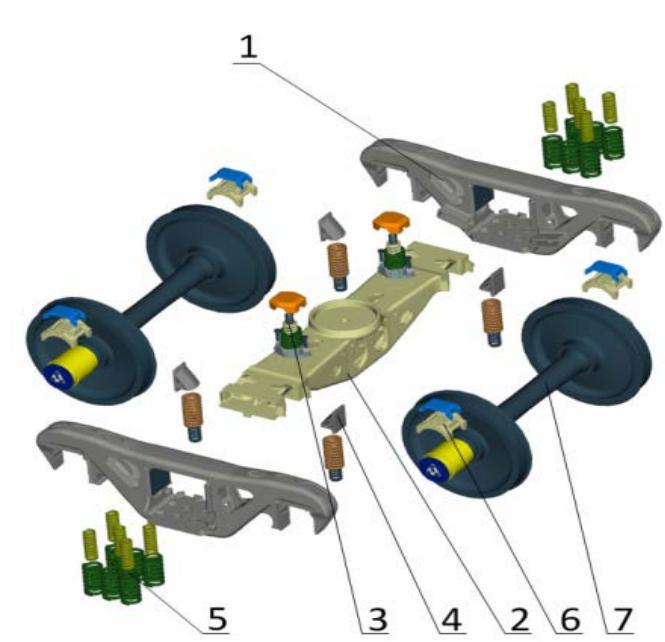

Figure 1. Three piece bogie "Motion control M975"Amsted rail, 1 side frames, 2 bolster, 3 side bearers, 4wedge, 5-suspension system, 6 elastic adapter, 7-wheelset

The connection between bolster and wedge has been described with one dimensional Saint Venant element, as it is shown in Figure 3, and connection between the wedge and the side frame has been described as two dimensional frictional Saint Venant element. The characteristics of Saint Venant element is possibility to introduce so called "stick-slip" movement into model [18].

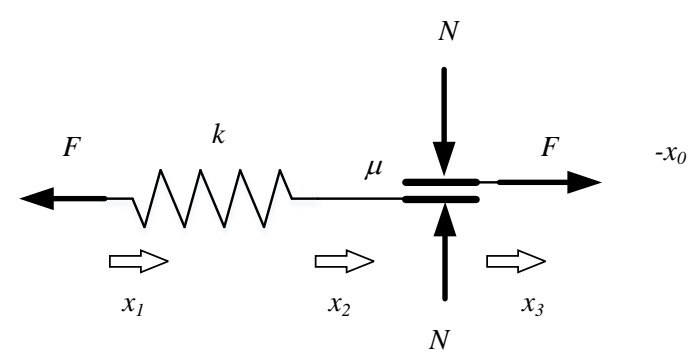

a)

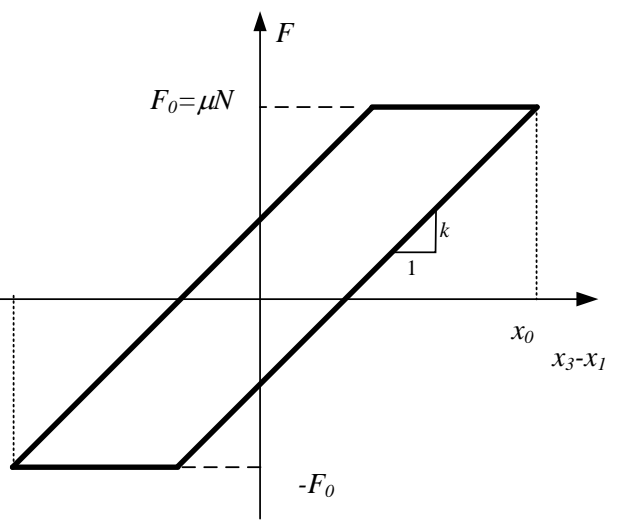

Figure 2. Saint-Venant element

Model of the Saint Venant element used in the vehicle model, b) Characteristic of the Saint- Venant element Force-displacement diagram

Taking into account the mass of the side frame and bolster, the mass of the wedge in the model can be neglected. 


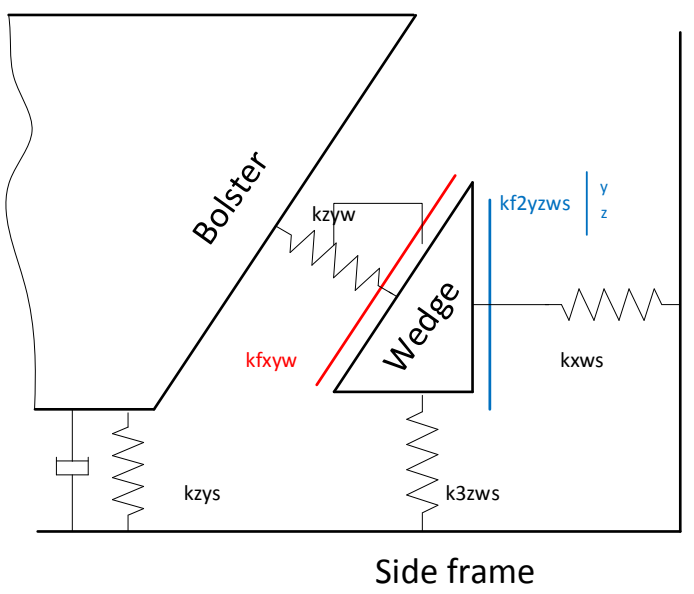

Figure 3. The suspension system model

The normal force which arises in the contact between bolster-wedge-side frames has been used for determination of the frictional force in Saint Venant element. The coefficient of friction, on the wedge sides cannot be exactly determined. Taking into account the excitation of the suspension system, the materials of the wedge, the bolster and the side frame, the value of the coefficient of friction has been estimated [7],[19].

The carbody has been connected to bogie over center plate and two side bearers, as it is shown in Figure 4. The side bearers are in constant contact with carbody, which continuously provide the friction force in longitudinal direction. The central plate provides the connection between the carbody and the bolster in vertical direction with possibility of rotation in yaw and sway direction. Due to carbody rotation around vertical axes, the frictional force arises in the side bearers, which is leading to better running stability of vehicle. In the case of the empty wagon, the side bearers are loaded with $80 \%$ of the carbody weight, and the rest of the weight is carried by the central plate. In the case of the loaded wagon, the central plate is loaded with $90 \%$ of the vertical load, while $10 \%$ is carried by the side bearers.

The model assumptions may be summarized as follows:

- Car body, bolster, side frames and wheels are modelled as rigid bodies,

- Side bearers have always contact with car body,

- Wedges are massless elements,

- Contact between the bolster and the wedge is a one dimensional friction block,

- Contact between wedge and side frame is a two dimensional friction block - in lateral and vertical direction,

- Adapter is modelled as rubber element with high stiffness in vertical direction,

- Clearances between elements are implemented in the model (bolster-side frame, axle-side frame, etc).

The model of the vehicle is developed in software package "Gensys".

\subsection{Railway track}

The model shown in Figure 4 takes into account vertical and horizontal stiffness of the track. By variation of the characteristics of the springs and the dampers in the model, tracks with wooden and concrete sleepers may be modelled, as well as different track stiffness, for example track stiffness during summer and winter ambient conditions [8].

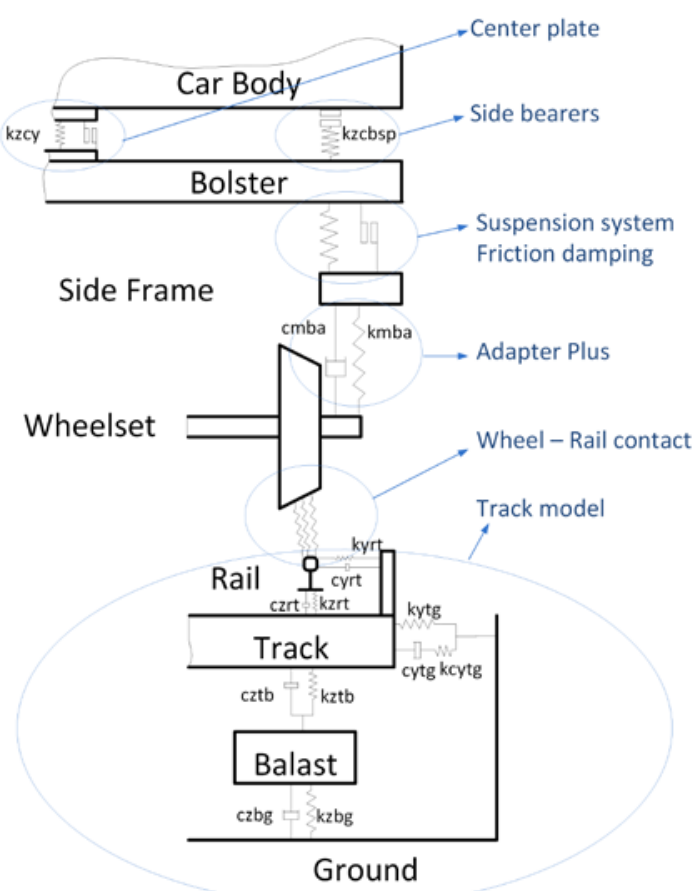

Figure 4. The model of the rail vehicle with three piece bogie

Since the wheelsets are exposed to increased load of 32t, wheel profiles WP4 especially optimized for highload and reduced wear, were developed.

During the vehicle negotiating the curve, due to centrifugal forces on the outside wheel, increased vertical and lateral forces arise. For this purpose, in the curves with small radius, outside rails have the optimized MB1 profile. On inner rail in the curve and on tangent tract rails have BV50 profile with inclination 1:30.

The MB1 profile on outside rail leads to decreasing of the wheel sliding over rail head and decreasing of the forces on outside wheel, which leads to decreasing of the wear of the wheels and rails in the curves.

The wheel-rail contact has been described by Kalker nonlinear theory [20] using kpfr functions in the software package „Gensys“. For measured profiles of the wheel and the rails, the equivalent conicity has been calculated.

Track geometry as well as track irregularities, such as longitudinal level, cant irregularity and gauge irregularity, have been recorded with measurement vehicle and introduced as excitation in vehicle dynamic simulations.

\section{THE EXPERIMENTAL INVESTIGATION OF DYNAMIC BEHAVIOUR OF VEHICLE}

Experimental investigations of the railway vehicle have been performed on commercial track, in north part of Sweden, between city Kiruna and port Luleå.

Experimental investigations have been performed during summer period with sunny days, on the dry track. On the basis of the weather conditions the value of 0.4 of the friction coefficient in the wheel-rail contact has been assumed. The coefficient of friction in the wheel-rail 
contact may have big influence in the calculation of the forces.

Measured gauge variations on the track parts chosen for model validation were $\pm 3 \mathrm{~mm}$. In simulations of empty and loaded vehicle the standard track gauge of $1435 \mathrm{~mm}$ has been assumed.

For simulation of the dynamic behaviour of the railway vehicle is assumed that vehicle is running with constant speed. The value of the vehicle speed has been calculated as the average value of real speed on each track part. The variations of the real speed was in the range of $\pm 0,4 \mathrm{~km} / \mathrm{h}$.

The accelerations in vertical and lateral directions have been measured with B12/200 inductive sensors. Measurement of the forces in wheel-rail contact has been performed with instrumented wheelset.

In the experimental investigation of the rail vehicle the following parameters have been measured:

- Accelerations above leading bogie, on the carbody floor, in vertical and horizontal plane,

- Accelerations in the middle of the carbody floor, in vertical and horizontal plane,

- Accelerations on side frame on leading bogie, in vertical direction,

- Vertical and lateral forces on wheels measured whit instrumented wheelset.

The instrumented wheelsets, produced by Interfleet, Stockholm, Sweden, have been installed on leading bogie. In this paper, for model validation, the following parameters have been compared:

- Accelerations above leading bogie, on the carbody floor, in vertical and horizontal plane,

The forces in the wheel-rail contact on leading wheelset in leading bogie, in horizontal and lateral directions.

\section{VALIDATION METRICS}

Model validation and verification are important steps in the process of the model developing. The selection of appropriate validation metrics is essential step in order to get an answer on the question "How good my model represent a real system?”. The well-known KolmogorovSmirnov metric and Area validation metric were been used for validation of the model of rail vehicle. Both validation metrics are based on comparison of the cumulative distribution functions (in future text denoted with CDF), but estimation of the signal similarity are based on the different approach.

\subsection{Kolmogorov - Smirnov test}

The two- dimensional Kolmogorov-Smirnov test is nonparametric test for equality of probability distribution functions. The maximal vertical distance between two CDF function is well known Kolmogorov-Smirnov metric. This metric mathematically may be expressed as following:

$$
d_{k \mathrm{~s}}=\sup \left|F_{1, n}(x)-F_{2, n^{\prime}}(x)\right|
$$

where $F_{1, n}$ and $F_{2, n}$ are two CDF from the signals obtained from simulation and from experiment which are compared. The graphical representation of the Kolmogorov-Smirnov distance is given in Figure 5.

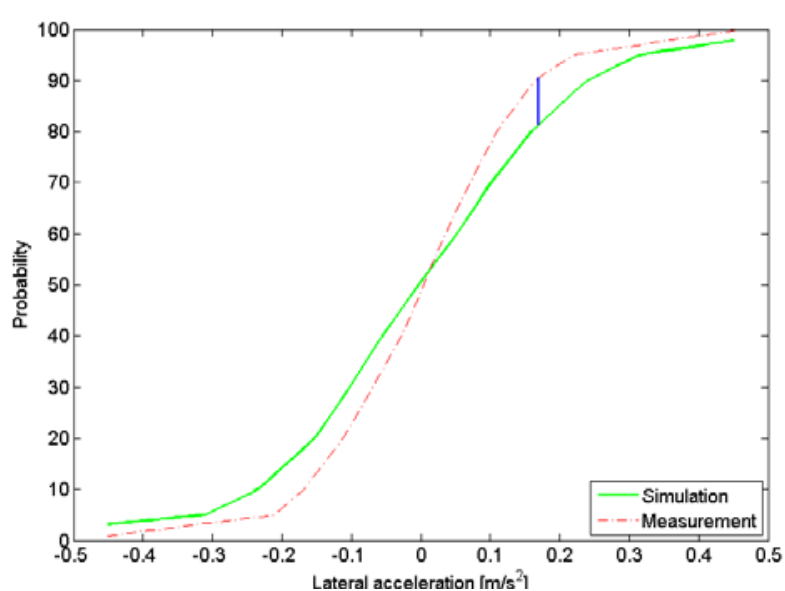

Figure 5. CDF function of simulation and measurement of lateral acceleration from simulation and experiment

Obtained K-S statistics is future is used for hypothesis testing where the null hypothesis is rejected at significance level $\alpha$ if a probability p has value which is greater than critical value.

The hypothesis are defined as following:

$H_{0}$ : the data in vectors $x 1$ and $x 2$ are from the same continuous distribution

$H_{1}$ : the data in vectors $x 1$ and $x 2$ are not from the same continuous distribution

The decision to reject the null hypothesis, in this paper, is based on comparing the p-value with the significance level $\alpha$, not by comparing the test statistic with a critical value.

The statistics defined in this manner does not describe the common underling distribution. Statistic only tests does two data sample have same distribution.

The Kolmogorov-Smirnov test can be modified to serve as a goodness of fit test. In the special case of testing for normality of the distribution, samples are standardized and compared with a standard normal distribution [16].

The main disadvantage of this validation approach is that estimation of the signal similarity has been based on difference of signals in one point. Only maximal difference between points in CDF's, which is defined by equation (1), while the resto of the cumulative functions are not considered.

\subsection{Area validation metrics}

Area validation metrics measure the difference between area of CDF of the simulation an experimental CDF, as it is shown in Figure 6. Mathematically, the area between two curves is the integral of the absolute value of the difference between CDF's:

$$
d_{a v}=\int_{-\infty}^{\infty}\left|F_{1, n}(x)-F_{2, n^{\prime}}(x)\right| d x
$$

or, in the case of the discrete functions area validation metrics may be expressed as:

$$
d_{a v}=\sum_{x=1}^{\infty}\left|F_{1, n}(x)-F_{2, n^{\prime}}(x)\right|
$$

Area validation metrics is a function of the shape of the distribution, but it is not readily interoperable as function of underlying random variable [15].

Figure 6 illustrates the area validation metrics as the mismatch between two CDF functions, where the 
difference has been denoted with gray colour. More about the area validation metrics may be found in literature [15].

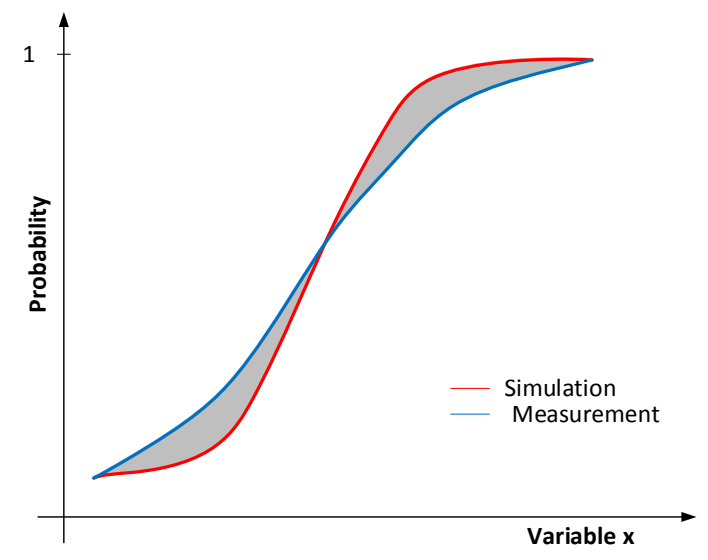

Figure 6. Example of the area validation metrics

In contrast to K-S test, as it is shown through equations (2) and (3) and in Figure 6, the area validation metrics estimation of the signal similarity is based on comparison of all points of CDF of two signals.

\section{RESULTS}

The simulations of the vehicle behaviour have been performed for the vehicle running in the curves with radius from $670 \mathrm{~m}$. The track profiles, curve radii, curve cant and track irregularities were measured and used as the input data for simulations. The length of the sections for estimation of the vehicle behaviour was chosen to be $250 \mathrm{~m}$, as it is shown in Figure 7.

Described metrics was applied for comparison of following parameters:

- Acceleration of carbody in lateral direction

- Acceleration on carbody in vertical direction

- Vertical force on outer wheel on leading direction

- Lateral force on outer wheel on leading wheelset

All signals, from simulation and from measurement, are filtered according to UIC recommendations [17]. On the basis of the signals in time domain the corresponding CDF functions are calculated and described metrics was used for model validation.

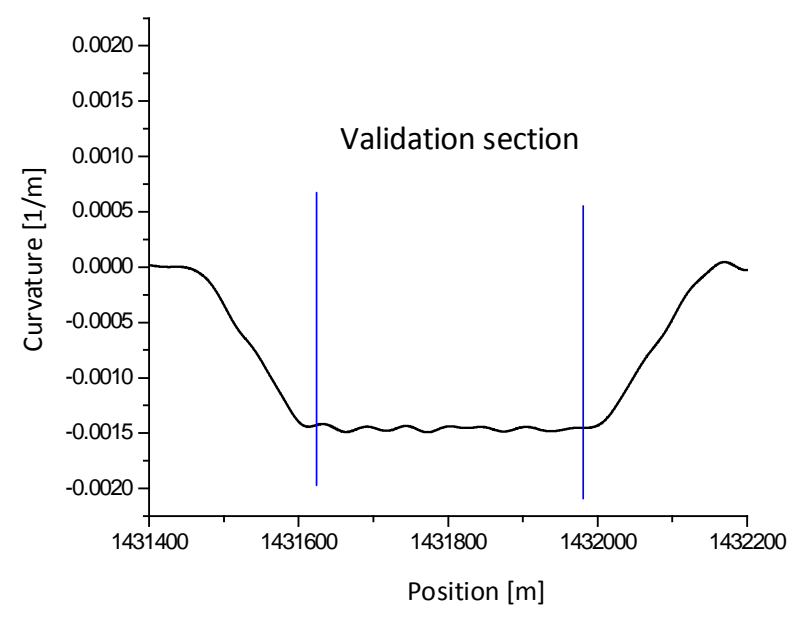

Figure 7. Section of track choosen for model validation
5.1. Acceleration of carbody in lateral direction

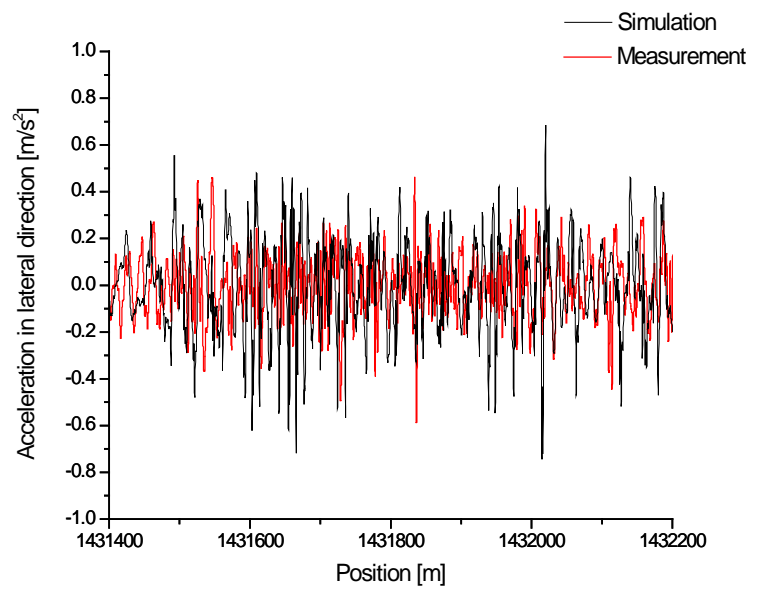

Figure 8. Acceleration of carbody in lateral directiontime domain

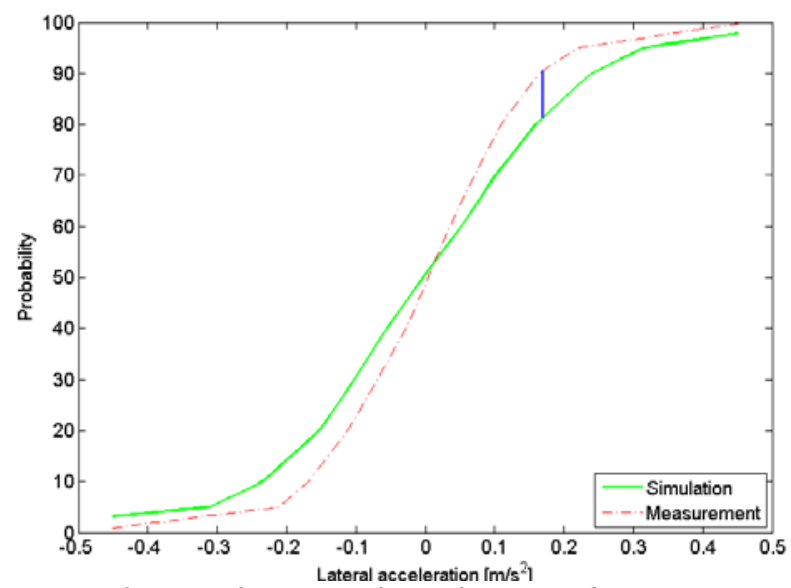

Figure 9. CDF function of simulation and measurement of lateral acceleration from simulation and experiment

Table 1. Results of comparison of lateral acceleration

\begin{tabular}{|l|l|}
\hline Metrics & Value \\
\hline K-S metric & 9.213 \\
\hline Area validation metrics & 9.591 \\
\hline
\end{tabular}

The CDF functions for lateral accelerations of the carbody obtained from simulation and experiment are described with 93 points (Figure 8). For two-tailed K-S hypothesis test, for significance level $\alpha=5 \%$ the probability value $p=0.2123$ which leads that null hypothesis is accepted. This mean that results from simulation and results obtained from experiment has the same distribution. 


\subsection{Lateral force on outer wheel}

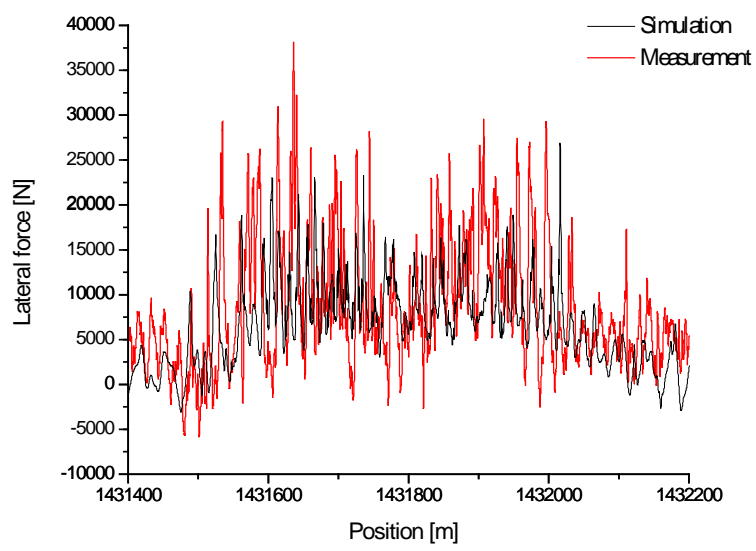

Figure 12. Lateral force on outer wheel - time domain



Figure 13. CDF function of simulation and measurement of lateral force on the outer wheel from simulation and experiment

Table 3. Results of comparison of the forces in lateral direction

Figure 11. CDF function of simulation and measurement of vertical acceleration from simulation and experiment

Table 2. Results of comparison of vertical acceleration

\begin{tabular}{|l|l|}
\hline Metrics & Value \\
\hline K-S metric & 3.9778 \\
\hline Area validation metrics & 15.4271 \\
\hline
\end{tabular}

The CDF functions for vertical accelerations of the carbody obtained from simulation and experiment are described with 141 points (Figure 11). For two-tailed K-S hypothesis test, for significance level $\alpha=5 \%$ the probability value $p=0.0579$ which lead that null hypothesis is accepted. This mean that results from simulation and results obtained from experiment has the same distribution.

\begin{tabular}{|l|l|}
\hline \multicolumn{2}{|c|}{ direction } \\
\hline Metrics & Value \\
\hline K-S metric & 27.3424 \\
\hline Area validation metrics & $1.4188 \times 10^{6}$ \\
\hline
\end{tabular}

The CDF functions for forces in lateral direction obtained from simulation and experiment are described with 391 points (Figure 13). For two-tailed K-S hypothesis test, for significance level $\alpha=5 \%$ the probability value $p=0.2361$ which lead that null hypothesis is accepted. This mean that results from simulation and results obtained from experiment has the same distribution. 
5.4. Vertical force on outer wheel

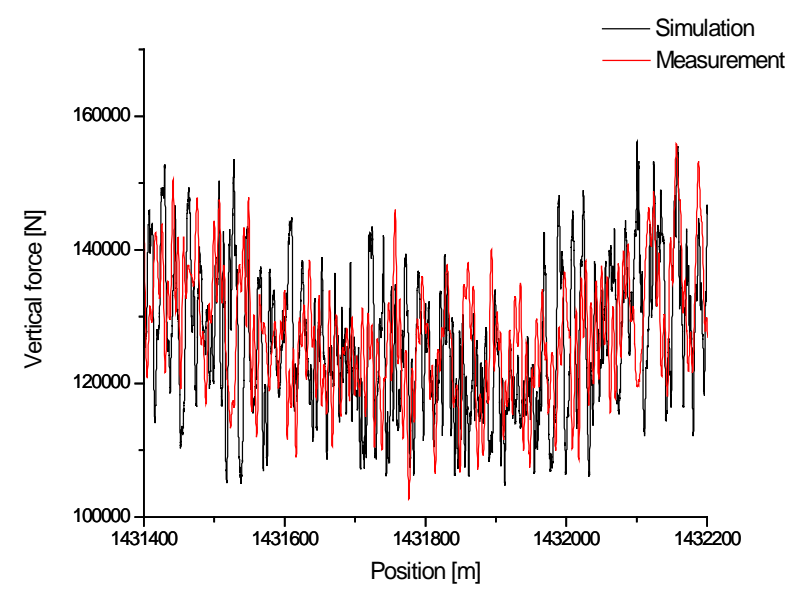

Figure 14. Vertical force on outer wheel - time domain

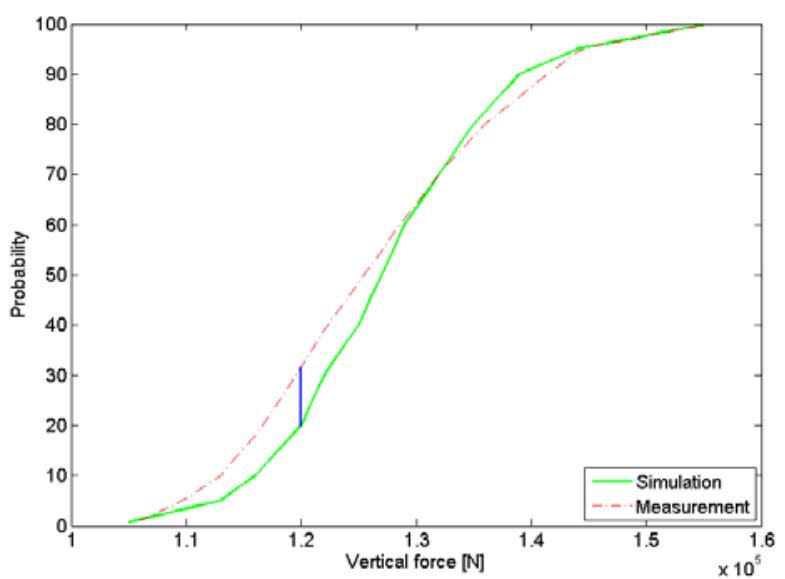

Figure 15. CDF function of simulation and measurement of vertical force on outer wheel from simulation and experiment

Table 4. Results of comparison of forces in vertical direction

\begin{tabular}{|l|l|}
\hline Metrics & Value \\
\hline K-S metric & 11.3501 \\
\hline Area validation metrics & $1.37 \times 10^{5}$ \\
\hline
\end{tabular}

The CDF functions for forces in vertical direction obtained from simulation and experiment are described with 56 points (Figure 10). For two-tailed K-S hypothesis test, for significance level $\alpha=5 \%$ the probability value $p=0.9649$ which lead that null hypothesis is accepted. This mean that results from simulation and results obtained from experiment has the same distribution.

\section{CONCLUSION}

The model validation has very important part in the model building and prediction of the behaviour of the mechanical systems. The nonlinear model of the freight rail vehicle where the real track condition and vehicle speed that are used as inputs for simulation of the vehicle behaviour, has been presented in this paper.

The Kolmogorov-Smirnov metric, Area validation metric and Hypothesis testing based on KolmogorovSmirnov metric, has been chosen for model validation.

The Kolmogorov-Smirnov metric show the maximum distance in vertical direction between two CDF functions. The limits for acceptance of the model has not clear or does not exist. For each of the considered parameters the limit for acceptance should be separately defined, which will make the process of validation more complicated.

The area validation metric is based on the difference of the area between two CDF's. The same conclusion as for the KS metric may be applied in this case. The limits, which will clearly define the acceptable model, do not exist.

From three presented metrics, the clearest conclusion about model acceptance may be drawn from hypothesis testing based on Kolmogorov-Smirnov metric. However, hypothesis testing does not give the answer how similar are two CDF function - two compared signals.

Overall, all three presented metrics do not give clear answer for validation of the presented model. The probability values $-p$ values from hypothesis testing looks promising and good starting point for development of new validation metrics for validation of the model of rail vehicles.

\section{ACKNOWLEDGMENT}

The authors wish to express their gratitude to Serbian Ministry of Education and Science for support through project TR37020.

\section{REFERENCES}

[1] Oberkampf, W. L., Trucano, T. G., and Hirsch, C. "Verification, Validation, and Predictive Capability in Computational Engineering and Physics,” Appl. Mech. Rev., 57(3), pp. 345-384, (2004)

[2] Sornette, D., Davis, A. B., Ide, K., Vixie, K. R., Pisarenko, V., and Kamm, J. R., “Algorithm for Model Validation: Theory and Applications,” Proc. Natl. Acad. Sci. U.S.A., 104(16), pp. 6562-6567, (2007)

[3] Ben H. Thacker, Scott W. Doebling, Francois M. Hemez, Mark C. Anderson, Jason E. Pepin, Edward A. Rodrigez, „Concepts of model verification and validation“, Los Alamos National Laboratory, Los Alamos, (2004)

[4] S.D. Iwnicki, A.H. Wickens, Validation of a MATLAB Railway Vehicle Simulation Using a Scale Roller Rig, Vehicle System Dynamics, Volume: 30, Issue: 3, Publisher: Citeseer, Pages: 257-270, ISSN: 00423114, DOI:10.1080/00423119808969451, (1998)

[5] Pelle Carlbom, Carbody and Passengers in Rail Vehicle Dynamics, Doctoral Thesys, TRITA - FKT 2000:48, ISSN 1103 - 470X, ISRN KTH/FKT/D--00/48-SE

[6] J.R. Evans \& P.J. Rogers "Validation of dynamic simulations of rail vehicles with friction damped y25 bogies”, Vehicle System Dynamics: International Journal of Vehicle Mechanics and Mobility, 29:S1, 219-233, http://dx.doi.org/10.1080/00423119808969561, (1998)

[7] Jönsson P-A, Persson I and Stichel S: New Simulation Model for Freight Wagons with UIC Link Suspension. Vehicle System Dynamics, Volume 46, Supplement, pp 695- 704, (2008).

[8] N. Bogojević, P.A. Jonnson and S. Stichel, "Iron Ore Transportation Wagon with Three-Piece Bogies - 
Simulation Model and Validation”, Proceedings of VII International Conference "Heavy Machinery HM 2011", Vrnjačka Banja (Serbia), 29 June-2 July 2011, pp. 33-38, (2011)

[9] Sprague, M. A., and Geers, T. L., “A Spectral-Element Method for Modelling Cavitation in Transient FluidStructure Interaction,” Int. J. Numer. Methods Eng., 15, pp. 2467-2499, (2004)

[10] Russell, D. M., "Error Measures for Comparing Transient Data: Part I, Development of a Comprehensive Error Measure," Proceedings of the 68th Shock and Vibration Symposium, Hunt Valley, MD, (1997)

[11] Russell, D. M., "Error Measures for Comparing Transient Data: Part II, Error Measures Case Study," Proceedings of the 68th Shock and Vibration Symposium, Hunt Valley, MD,( 1997)

[12] H. Sarin, M. Kokkolaras, G. Hulbert, P. Papalambros, S. Barbat and R.J. Yang, "A comprehensive metric for comparing time histories in validation of simulation models on emphasis on vehicle safety applications", Proceedings of the ASME 2008 International Design Engineering Technical Conference and Computers and Information in Engineering Conference, (2009)

[13] A. Kolmogoroff, "Confindence limits for an unknown distribution function”, Ann. Mathh. Statist. 12 461-463, (1941)

[14] Anderson, T. W.; Darling, D. A. "Asymptotic theory of certain "goodness-of-fit" criteria based on stochastic processes". Annals of Mathematical Statistics 23: 193212. doi:10.1214/aoms/1177729437, (1952).

[15] Scott Ferson, William L. Oberkampf, Lev Ginzburg, "Model validation and predictive capability for the thermal challenge problem”, Comput. Methods Appl. Mech. Engrg. 197 2408-2430, (2008)

[16] http://www.princeton.edu/ achaney/tmve/wiki100k/d ocs/Kolmogorov-Smirnov_test.html

[17] UIC testing and approval of railway vehicles from the point of view of their dynamic behaviour -safety- Track fatigue - Ride quality, Code 518 OR, $4^{\text {th }}$ edition, Paris, (October 2009).

[18] B. D. Yang, M. L. Chu And C. H. Menq, Stick-slipseparation analysis and non-linear stiffness and damping characterization of friction contacts having variable normal load, Journal of Sound and Vibration 210 (4), 461-481, (1998).

[19] A. Orlova \& Yu. Romen "Refining the wedge friction damper of threepiece freight bogies”, Vehicle System Dynamics: International Journal of Vehicle Mechanics and Mobility, 46:S1, 445-455, (2008).

J.J. Kalker, Wheel-rail rolling contact theory, Wear, Volume 144, Issues 1-2, Pages 243-261, ISSN 00431648, http://dx.doi.org/10.1016/0043-1648(91)90018-P, (1991) 